\section{\$4. Effect of Air on Energy and Rise-Time Spectra Measured by Proportional Gas Counter}

Kawano, T., Tanaka, M.,

Isozumi, Y., Tosaki, M. (RRC. Kyoto Univ.),

Sugiyama, T. (Nagoya Univ.)

Tritium emits beta-rays following respective disintegrations. The maximum and average energies of beta-rays are 18.6 and $5.7 \mathrm{keV}$, respectively. As the energy of beta rays is very low, how to discriminate tritium signals from all other noise signals is a key point for measuring tritium. In the case of measuring tritium concentration in the exhaust air, the tritium gas monitor is often used. A typical gas monitor is a direct-reading device that is installed a vented gas chamber and able to continuously measure the tritium concentration in flowing air. However, commercially available conventional tritium gas monitor does not have sufficient detection capability to detect the legal limit of tritium concentration in air because the legal concentration limit for tritium in air is very low compared with the tritium sensitivity of a conventional gas monitor.

Overcoming this situation, the development of gas monitor dedicated to measuring tritium in air was started. In the monitor currently under development, a proportional gas counter is employed because the proportional gas counter arise pulse signals corresponding to incident radiation energy. In the case of tritium, the signal pulses have very low pulse height with very fast rise-time. Using the features of low pulse height and fast rise-time, true tritium signals could be discriminated from all other noise signals due to incident gamma rays from external sources such as cosmic radiation.

In actual monitoring, a proper amount of air sample is mixed in counting gas beforehand and the tritium concentration contained in the air sample is measured using the mixture of counting gas and air sample gas as counting gas of the counter. It is unfortunate that air contains oxygen and water vapor that have high electron attachment coefficient in air, because those elements trap electrons from electron-ion pairs created by ionization arose by beta rays emitted from tritium contained in sample air. This electron trapping causes depletion of number of electrons and results in a reduction of beta ray counts. To examine the effect of air, energy and rise-time spectra were measured using the mixture of counting gas (methane) and air $(2.5,5.0$ and $7.5 \%)$ dried through a silica gel column using an unsealed ${ }^{55} \mathrm{Fe}$ radiation source. The unsealed ${ }^{55} \mathrm{Fe}$ radiation source emits an auger electron with energy of 5.9 $\mathrm{keV}$, corresponding to the average energy of beta rays emitted from tritium.

In Fig. 1 energy spectra obtained are shown and it is found that the width of peak becomes narrower moving to the lower energy channel as a proportion of air increases and more than $10 \%$ air may bring about the disappear of spectrum. As peak positions mean energy of electron detected, the movement of peak positions to lower energy side might suggest an occurrence of electron trapping by oxygen in air, because nitrogen has a low electron attachment coefficient in air.

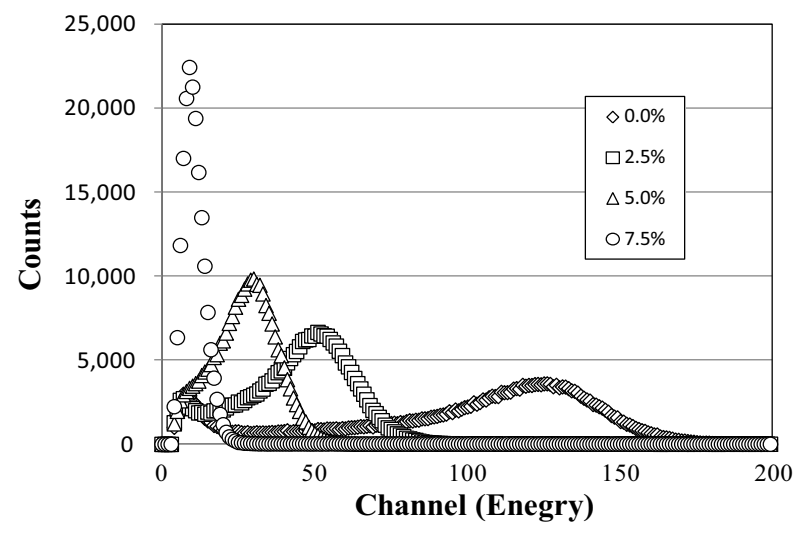

Fig. 1 Effect of sample air mixed in counting gas on energy spectra

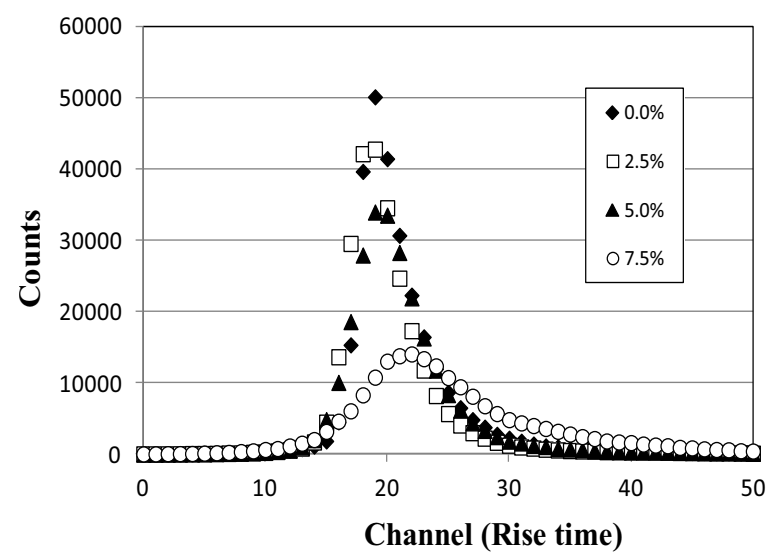

Fig.2 Effect of sample air mixed in counting gas on rise-time spectra

In Fig.2, rise-time spectra are shown. It is also found that the width of peak becomes narrower moving to the lower rise-time channel as a proportion of air increases, but not so significant compared with those in energy spectra. This result suggests that the rise-time spectra might be more tolerate than the energy spectra for effect of air. If using rise-time spectra, tritium concentration measurement may be possible for more rich air contained gases. It could be determined that air will degrade the performance of the detector and future detail studies are necessary for clearing the mechanism of the movement of peak position by the effect of air, 